\title{
Processi e fenomeni di discriminazione e de- umanizzazione dal secolo scorso ad oggi
}

Appunti per una storia culturale del razzismo nella pratica didattica

di Enrico Manera

Feb 16, 2021 | Emergenza e (nuova) normalità, In evidenza, Uguaglianza/Differenza | $\underline{0}$ |

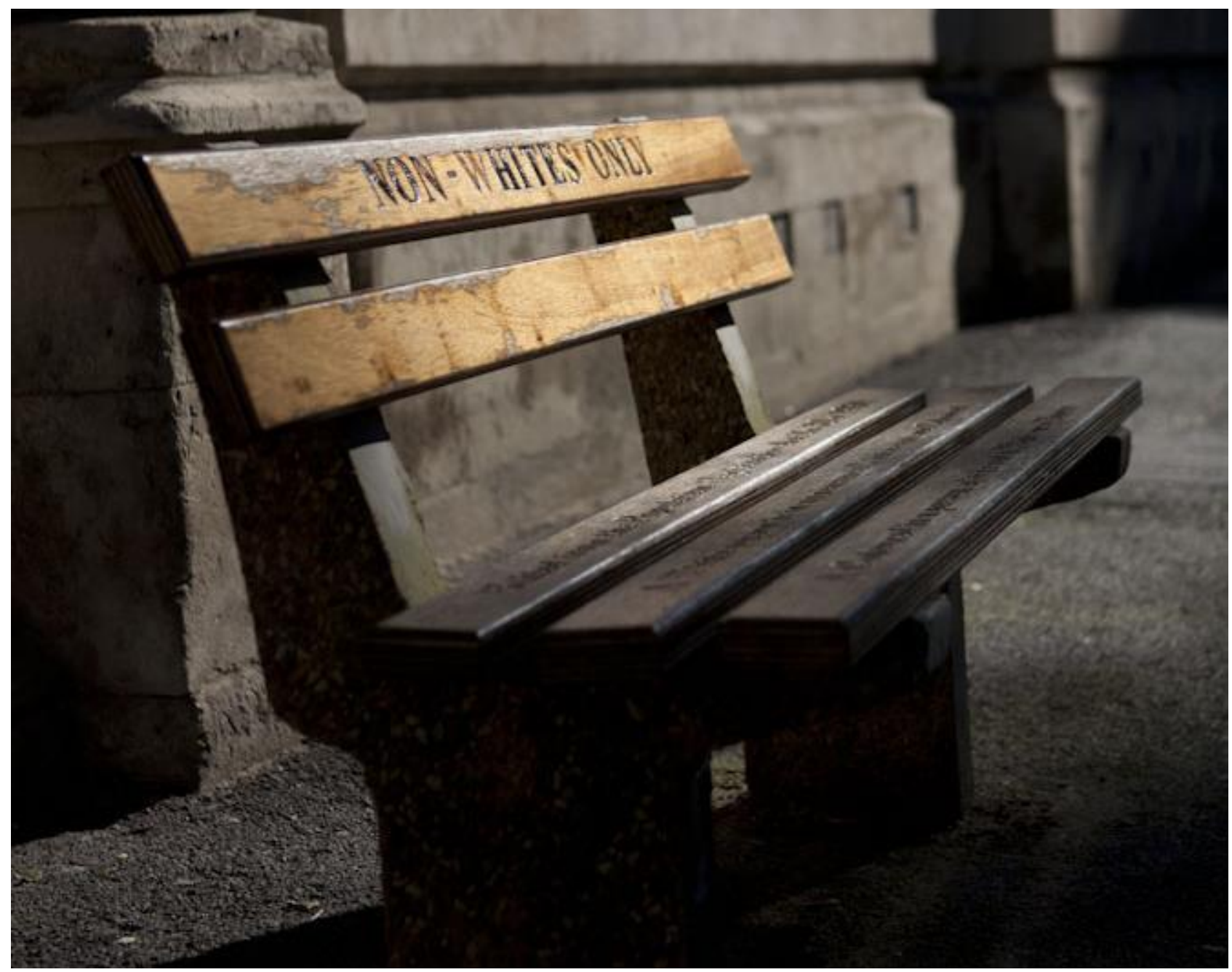

Non-whites only apartheid bench (opera artistica), Città del capo, Sudafrica.

Foto di Hannah Mentz - Own work, CC BY-SA 3.0, Link

Il dossier sulla Summer school 2020

si compone di 5 coppie di parole chiave.

Questo testo afferisce alla coppia di parole chiave

Uguaglianza/Differenza 


\begin{abstract}
Nella seconda giornata della Summer School 2020 abbiamo chiesto a Enrico Manera e Chiara Volpato di riflettere con noi sulle parole chiave Uguaglianza e Differenza. L'intervento di Manera, di cui il testo che segue è una traccia rivista dall'autore, ricostruisce a fini didattici alcuni spunti teorici, le principali manifestazioni di discriminazione e de-umanizzazione (di razzismo) che si sono verificate nella storia europea e suggerisce alcuni spunti di analisi del presente.
\end{abstract}

\title{
Identificazione ed essenzialismo
}

All'interno delle società umane sono diversi i processi che determinano quei fenomeni di identificazione, etnogenesi e definizione delle soggettività che possono generare il razzismo. Parlare di questi processi e del "come" essi si alimentano significa mettere a fuoco innanzitutto che cosa intendiamo quando diciamo identità e quindi, in estrema sintesi, la dimensione storica ed artificiale dei processi di identificazione politica.

La nozione di identità è centrale per comprendere quella di discriminazione. Essere "identico a", la coincidenza, implica essere "diverso da", la differenza. In questo senso il sentimento di appartenenza che viene sintetizzato come identità - qualunque essa sia - si determina continuamente in base a processi di reciproca differenziazione, ovvero nel riconoscimento dell'altro come non-sé: si può riconoscere un io solo in riferimento all'altro e nel flusso del tempo. Il rinvio all'altro, lo scarto, è la condizione dell'identità, ed è dunque quello che non si vede quando diciamo "identità", ovvero la relazione che istituisce l'autocoscienza dei soggetti. Se la differenza viene posta come un limite insuperabile, un dato immodificabile o un problema, prendono vita, in determinate condizioni storiche, processi di discriminazione e di de-umanizzazione.

Questi rendono possibile l'esercizio della violenza nei confronti di altre persone cui viene negato lo status di umanità. Dire "chi siamo" implica sempre dire "chi non siamo", ovvero le forme distanti da noi, che molte volte ha implicato la disumanizzazione dell'altro. È ormai vastissima la produzione sul tema[1]: a partire dallo studio dei genocidi l' "invenzione" dell'altro è una condizione della violenza, in base all'idea che un gruppo esista e si identifichi in sé solidamente producendo una forma di odio che viene proiettato in funzione coesiva su un gruppo-vittima; in questo senso lebbrosi, ebrei, minoranze, devianti sessuali ma anche donne - oggetto di specifica persecuzione nella storia - costituiscono diverse declinazioni di un'identica matrice. Sintetizzando un tema che è stato affrontato da Francesco Remotti e dalla sua scuola[2], l'identità è una finzione, un costrutto artificiale, non è qualcosa di sostanziale, ma un sentimento di appartenenza divenuta riflessiva e socialmente costruito. In altri termini, è un mito[3]. L'identità dunque non esiste, non è una cosa, e in ogni caso non può essere considerata una né fissa, ma è il nome improprio assegnato storicamente a un intrico di sentimenti soggetti a storicità. Si tratta di un senso di appartenenza che si sostanzia in alcune prassi e che solo un'errata concezione logica può considerare al pari di una sostanza. Questo è il presupposto logico su cui si costruisce il razzismo, ovvero ogni dottrina secondo la quale gli individui di un gruppo nascono portatori di una data cultura e sono soggetti a un dato destino, concepito come un set standard di caratteri biologici, morali e culturali non modificabili, ordinati gerarchicamente[4].

Una volta che si è supposta l'esistenza di un'identità, tale rete dinamica di relazioni riflessive, estrapolata dal contesto idealtipico e privata del suo carattere mobile, viene ridotta e semplificata per poi essere utilizzata consapevolmente come una nozione escludente e stigmatizzante.

Nei processi di identificazioni i soggetti stessi sono attivi, il loro valore di credenza forte è in grado di orientare le azioni e le decisioni. Le identità sono creazione di fiction, manipolabili e strumentalizzalibili nei diversi contesti del loro utilizzo, nei quali la dimensione politica è decisiva: 
le identità sono soprattutto progetti politici, preparati e strategicamente condotti da élite determinate al conseguimento di un risultato. Sono fenomeni storicamente fondati sulla lotta per il potere tra gruppi rivali a livello sociale, culturale e politico[5]. Un'identità è di fatto il risultato di un insieme di elementi culturali entrati nei processi di socializzazione e che guidano le credenze degli individui che vi si riconoscono. Come insegna la storiografia più avvertita, ogni processo di State Building non coincide con quello di Nation Building. Il processo di la «nazionalizzazione delle masse» di cui ha parlato Georg Mosse[6] descrive quelle pratiche di socializzazione, acculturazione, educazione attraverso le quali in età contemporanea $\mathrm{i}$ cittadini sono stato indotti a assoggettarsi alle regole e ai quadri sociali della memoria culturale e pubblica in cui erano immersi, orientata in senso di un culto religioso della nazione e della patria.

Da un punto di vista teorico, la cultura non è una realtà di tipo super-organico rigida e definita: al contrario risulta dinamica, fluida, mutevole all'interno di rapporti di continua negoziazione che $i$ diversi soggetti intrattengono con sé e tra di loro all'interno delle cornici culturali.

Da un punto di vista storico, l'individuo non è sovradeterminato dalla cultura e le società non sono mai mono-culturali, in nessuna epoca fin dai tempi più antichi, e si sono invece costruite sullo scambio, sull' incrocio e sull'osmosi, nell' incontro come nel conflitto[7]. Lingua, cultura e identità sono sempre state al centro di veri e propri progetti politici e di strategie discorsive radicate nella storia: le identità, appartenenze nazionali e regionali, etniche o religiose, sono dunque concetti problematici che andrebbero trattati con molta accortezza, soprattutto quando si insegna storia o le si maneggia in pubblico e nel discorso politico.

Un tale discorso complesso manca non solo a livello diffuso nell'opinione pubblica ma anche nella divulgazione quando non nella manualistica scolastica, ancora legate a modelli teorici impliciti, essenzialisti e sostanzialisti, e a pregiudizi storiografici otto-novecenteschi, che sono in definitiva racconti mitici di un'autonarrazione degli stati nazionali.

\section{Esclusione e razzismo nel Novecento}

Parlare di razzismo ed esclusione nel Novecento richiede un ragionamento di più lunga durata capace di mettere a fuoco alcuni momenti nella storia europea per giungere fino alla violenza nazista nella Seconda guerra mondiale, intesa come culmine della guerra europea dei Trent'anni del Novecento, all'interno del totalitarismo e sullo scenario della guerra totale[8].

Sono infatti diverse e numerose le forme di distinzione e discriminazione nella storia, che diventano forme di razzismo. A partire dalle dinamiche di xenofobia note al mondo antico si giunge fino alla razzializzazione che in età moderna vede sorgere le categorie stesse di razza, etnia e cultura. Si tratta di forme concettuali di organizzazione del vivente che "sono servite" per definire e costruire le relazioni con soggetti altri, e che in particolare assumono tratti di separazione, odio e violenza quando si rivolgono a minoranze e subalterni.

Il razzismo non è semplicemente un pregiudizio o un sentimento negativo ma è una forma di sapere che in determinati momenti storici si istituzionalizza come forma di legittimazione del dominio e in questo assolve una funzione mitica centrale per la stabilizzazione di uno o più poteri nei gruppi umani.

Il pensiero europeo-occidentale ha organizzato, catalogato e costruito rapporti, sostanzialmente di dominio, che si intersecano con le geometrie variabili della classizzazione e della 
sottoproletarizzazione dei gruppi; ovvero la loro subordinazione gerarchica in termini di accesso alle risorse e ruolo all'interno delle società[9].

In modo schematico, è possibile individuare una tendenza di origine cognitiva a dividere, classificare e semplificare le variabili possibili dell'esistenza, su cui si innesta l'organizzazione di potere e la logica di subordinazione, dentro le quali l'immaginario di distinzione legittima le forme di esercizio del potere e della violenza. Da un punto di vista didattico è fondamentale mettere a fuoco la storia del razzismo ma anche retrodatarla lungo l'asse del tempo.

Non è possibile in questo contesto fare una completa storia culturale del razzismo, ci sono però diversi momenti paradigmatici che nella storia - in particolare quella che facciamo a scuola possono essere evidenziati per la sensibilizzazione al tema della violenza razzista e genocidaria e che da un punto di vista geneaologico anticipano e preparano le successive logiche di disumanizzazione.

Se concentriamo la nostra attenzione sulla storia contemporanea, il senso di appartenenza territoriale e le logiche del "sangue" - discendenza, parentela, tradizione - con relative forme di xenofobia si modificano nel tempo in relazione alla nascita e alla reinterpretazione dei nazionalismi dell'Ottocento. Come ha mostrato Contini nel 1959, razza è un termine che dalle prime attestazioni in italiano antico si diffonde alle lingue europee ed è una trasformazione medievale dell'antico francese haraz, che indica un allevamento di cavalli, una mandria, un branco: ha «una nascita zoologica, veterinaria, equina» (Contini)[10] e un'appartenenza alla sfera semantica dell'animalità.

Dalla seconda metà dell'Ottocento la riflessione europea sulla differenza, una vera e propria razziologia, si associa e si innesta sul discorso nazionalista, che vede nel sangue e nella comunità nazionale due idee chiave. A partire dagli anni Settanta vengono allestiti zoo umani, imparentati con giardini zoologici ed esposizioni universali, in cui abitanti di diverse aree del pianeta, in particolare "selvaggi", sono animalizzati, tenuti in gabbia e in scenari "naturalistici" ricostruiti e inseriti in una narrazione evoluzionistica che li inscrive nel mondo dei primitivi. Nativi africani, australiani o fuegini erano considerati residui preistorici, veri e propri "fossili viventi" relegati ai margini del globo, il cui presente sarebbe il passato europeo; l'avversione, talvolta patologica, nei confronti dell'Africa o del primitivo si collega anche al rifiuto dell'evoluzionismo e al trauma culturale che il darwinismo introduce nella cultura europea e che nel giro di poco subirà un'interpretazione sociale, contro le stesse intuizioni e intenzioni di Darwin. Tali idee hanno poi goduto di una incredibile fortuna nel Novecento e hanno caratterizzato molte idee "antropologiche" - oggi falsificate - ma ancora solidamente diffuse grazie anche alla religionistica di impianto eurocentrico e all'esotismo orientalistico. Mentre l'antropologia, che nasce positivista e coloniale, è stata una delle prime discipline a tematizzare il problema della sua origine, a storicizzare e a decostruire i propri saperi[11], molta filosofia, critica letteraria e storia delle religioni di marca metafisica risultano problematiche nel lungo periodo e in forza della loro tradizione e scholarship, come mostrano le ricorrenti polemiche su figure anche importanti e rispettate (esempi eclatanti sono Heidegger e Eliade), che nella prima metà del Novecento non solo hanno militato in organizzazioni di estrema destra o sono stati legati a regimi totalitari, ma nel loro campo disciplinare e dal punto di vista teorico hanno avallato idee metastoriche, razziste e pregiudizi insostenibili.

Il razzismo ottocentesco e il darwinismo sociale che accompagnano l'epoca positivista aggiungono alla classificazione un'esplicita gerarchizzazione con la definizione di una scala, al cui vertice si colloca la "razza bianca", fatta coincidere con buona approssimazione con la condizione occidentale nord-europea. I fondamenti teorici di grande influenza possono essere individuati in opere molto diffuse, sulla base di studiosi come Müller o Renan, che ereditano la cultura illuminista di Linneo e 
di Kant, o quella romantica degli Schlegel o degli Schopenhauer, per poi arrivare ai razziologi veri e propri del XIX secolo, come de Gobinau, Galton, Vacher de la Pouge, Chamberlain.

È a partire da questa base culturale che verranno rielaborate le teorie eugenetiche e razziste che fanno da sfondo alle imprese coloniali dell'Ottocento e del Novecento, con i loro correlati di violenza estrema, ed a questi che dobbiamo guardare per comprendere più a fondo il rapporto con il totalitarismo nazista e quello italiano nella Seconda guerra mondiale.

La mondializzazione attuale e i tanti conflitti del presente, in connessione con il passato moderno, sono elementi ulteriori che inducono al considerare come la violenza razzista e coloniale siano $\mathrm{i}$ prodromi della violenza politica di massa del Novecento. La colonia è il luogo di incubazione dell'internamento dei civili e degli eccidi di massa che troveranno il loro apice nel sistema concentrazionario e sterminazionista nazista nel cuore della Seconda guerra mondiale[12]. In termini schematici, utili per gli insegnanti impegnati a delineare uno scenario di fondo in un progetto educativo, si tratta di guardare al "lungo Ottocento" per individuare nel colonialismo gli antefatti di una violenza che, in altri contesti, detona poi in Europa, a partire dalla Grande guerra. Le pratiche della violenza efferata e industriale della Shoah con il loro tratto inedito sono le estreme conseguenze della sintesi di darwinismo sociale, ideologie reazionarie e imperialismo aggressivo che il Terzo Reich e i suoi alleati sono stati capaci di realizzare nell'Europa moderna[13].

\section{I volti più recenti del razzismo}

Guardando al presente in termini di sociologia storica si può evidenziare un modello concettuale per cui ogni crisi culturale, economica, di sistema implica una chiusura dei gruppi sociali maggioritari in senso identitario e omogenizzante e provoca l'attivazione di pratiche di esclusione su gruppi minoritari già discriminati, rispetto ai quali esiste un repertorio di stereotipi negativi[14].

Anche all'interno delle società democratiche un presunto privilegio, e la supposta responsabilità in un periodo di crisi del soggetto definito e vissuto come diverso, diventano il soggetto di fantasie proiettive e vittimismitiche di segmenti di popolazione che vedono un peggioramento della propria condizione e cercano sicurezze e riferimenti politici rassicuranti e capaci di promettere un "ritorno" all'ordine alle stabilità di periodi precedenti.

Sullo scenario di una mondializzazione sempre più complessa, credenze diffuse generate dalla paura dell'altro sono miti operativi che alimentano tensioni e rancori di natura sociale ed economica. La sistematica demonizzazione del diverso legittima innanzitutto l'esclusione. La definizione dell'immagine "chiusa" dell'altro è funzionale alla determinazione della propria identità comunitaria/nazionale/etnica e può mettere in moto fenomeni reattivi di esclusione ulteriore.

Lo studio comparativo dei genocidi, in diversi contesti di crisi, ha messo in luce, alla radice della violenza, l'«ideologia della differenza assoluta tra il Sé (posto come bene) e l'Altro (posto come male)»[15]; la forma mentis che produce/stabilizza le identità procede per differenziazione e separazione: se/quando diventa ossessione della purezza e timore del contagio, la sistematica demonizzazione del diverso legittima l'esclusione. Questa è il primo livello di una escalation dell'odio le cui tappe progressive - marginalità, segregazione, deportazione, sterminio - sono storicamente interconnesse, benché non deterministicamente correlate. 
I temi della discriminazione e della de-umanizzazione ricoprono grande importanza per l'insegnamento dell'educazione alla cittadinanza - ora formalizzata come Educazione civica -, e sono tanto più importanti quanto più quanto tali pratiche si ritrovano presenti in modo esplicito e drammatico nelle culture autoritario-populiste e post-fasciste del presente, in un tempo in cui patenti violazioni dei diritti umani convivono con le democrazie europee e passano sotto la soglia di percezione dell'opinione pubblica, o addirittura sono consapevolmente accettati. Gli stereotipi nazionalisti, antisemiti, xenofobi, l'immaginario demonizzante e criminalizzante sui migranti sono oggi in Europa, e non solo, immagini semplificate della realtà, miti identitari che danno risposte facili a crisi di drammatica intensità e complessità[16]. I "nuovi" volti del razzismo non sono affatto nuovi, perché si riconnettono a precedenti politici e a tradizioni consolidate considerate come referenti (anche simbolici), concretizzati in luoghi comuni e immagini di lunga durata e sopravvivenza, radicate e diffuse nelle culture nazionali, in particolare dei paesi che abbiano vissuto regimi dittatoriali.

In contesti di democrazia in crisi, come quello italiano, è possibile attestare una proliferazione di culture di destra, non separabile dalla ripresa populista e sovranista di riferimenti o allusioni alle pratiche autoritarie del fascismo (quando non del nazismo). Si tratta di culture di grande reviviscenza nella sfera mediatica e nei contenuti di larga diffusione, che propongono una riformulazione attualizzante di rapporti, relazioni e prassi politiche volte al futuro, su scenari che fino a poco tempo si sarebbero detti distopici[17]. La novità consiste dunque in un consapevole sfruttamento di temi identitari da parte della politica istituzionale nell'agone democratico e di un più ampio coinvolgimento della produzione di idee "dal basso" con significativi slittamenti concettuali e sociali: gli identarismi sono forme sofisticate di differenzialismo culturale, stanno alla base delle teorie degli ideologi delle nuove destre radicali e sono giunte alla presentabilità e alla visibilità attraverso strategie di conquista dell'egemonia culturale, spesso anche con titoli universitari e pubblicazioni che trovano accoglienza negli ambienti neoliberali e nell'opinione pubblica più "moderata".

Dietro parole diverse come etnicità e cultura il riferimento è però la più semplice e triviale "razza", diversamente argomentata, nascosta e depurata dagli aspetti più sgraditi alle vuote retoriche democratiche. C'è stato uno scivolamento del razzismo dalla "razza" a quello dell'"etnia" e della "cultura", in direzione di una forma di avversione per il diverso da sé in versione bio-storica o etnico-culturalista o identitaria, che dir si voglia. Nella storia del presente le presunte identità etniche, culturale (e per certi versi religiose) sono dunque versioni post-moderne e più recenti del razzismo, utili a preservare privilegi o diritti pensati come beni cui limitare l'accesso ai soggetti che si percepiscono come nativi e quindi oggetto di attenzione prioritaria. Come la "razza", identità, culture, etnie e provenienze sono pensate e percepite come entità fisse e destorificate: il loro esito ultimo risulta la mancanza di disponibilità ad accettare le differenze e il rifiuto della solidarietà (se non il disprezzo dei diritti umani)[18]. Antropologie "fantasma" di tipo razzializzante, reti discorsive conservatrici di ampia estensione, immaginari post-fascisti o neo-fascistoidi producono dunque versioni mitiche della realtà che impediscono di vedere come i migranti di ogni condizione sia trattati come non persone, oggetto di esclusione, indifferenza e violenza sistematica, come avviene nei campi in Libia e ai confini dell'Unione Europea, luoghi di sospensione del diritto e dell'umanità a cui di fatto è stato affidato il compito di filtrare e impedire i movimenti di persone verso l'Europa[19]. Non può non colpire come tali idee circolino massivamente nell'opinione pubblica e sui principali organi di informazione, caratterizzati un livello gravemente deficitario di analisi, e abbiano cittadinanza in particolare nel web e nel mondo dei social media, dove appaiono inoltre come "narrazioni controcorrente", contro un presunto "pensiero unico" o contro la "dittatura del politicamente corretto". Il neo-razzismo, una pratica diffusa e legittimata anche da personalità e partiti con ruoli e responsabilità di governo, si maschera e si giustifica dietro nuove retoriche di insorgenza gentista e vittimismo populista-localista; un fenomeno che ha contribuito alla 
normalizzazione del discorso su razzismi e fascismi, come fossero dati inevitabili da accettare e di cui prendere atto[20].

Ancora una volta la semplificazione della realtà e la negazione della complessità dell'esistenza, a partire dalla mancata conoscenza elementare di fatti e processi storici, possono aprire la via all'affermazione di realtà politiche antidemocratiche e antipluraliste, autoritarie e violente. Tra le emergenze del presente e tra i compiti di un sapere storico che abbia valore civile figura la costruzione di un nuovo racconto dell'antropologia e dei rapporti culturali in nome di un rinnovato patto di cittadinanza, che sia anche capace di ampliare lo sguardo della riflessione storica pubblica.[21]

Note:

[1] T. Todorov, La conquista dell'America. Il problema dell'"altro", Einaudi, Torino 1982; P. Levi, I sommersi e i salvati, Einaudi Torino, 1986; G. Bensoussans Genocidio. Una passione europea, Marsilio, Venezia 2006

[2] Cfr. F. Remotti, Contro l'identità, Laterza, Roma-Bari, 1996

[3] Cfr. J. Assmann, La memoria culturale, Einaudi, Torino 1997

[4] F. Jesi, Cultura di destra, Garzanti, Milano, 1979.

[5] M. Aime, Eccessi di culture, Einaudi, Torino, 2004.

[6] G. Mosse, La nazionalizzazaione delle masse, Il mulino, Bologna, 1975.

[7] Cfr. G. Leghissa e D. Zoletto (a cura di), Gli equivoci del multiculturalismo, «aut aut »312, novembre dicembre 2002.

[8] E. Traverso, A ferro e fuoco, la guerra civile europea, Bologna, il Mulino 2007 ; J. Chapoutot, La rivoluzione culturale nazista, Laterza, Roma-Bari, 2019.

[9] M. Aime, Classificare, separare, escludere. Razzismi e identità, Einaudi, Torino, 2020.

[10] G. Contini, I più antichi esempî di «razza» in «Studi di filologia italiana», XVII, 1959.

[11] C. King, La riscoperta dell'umanità. Come un gruppo di antropologi ribelli reinventò le idee di razza, sesso e genere nel XX secolo, Einaudi, Torino, 2020.

[12] C. Vercelli, Il dominio del terrore. Deportazioni, migrazioni forzate e stermini nel Novecento, Salerno, Roma, 2015.

[13] F. Soverina, La violenza politica di massa. Guerre, stermini e genocidi nella prima parte del Novecento, in Resist-oria. Bollettino dell'Istituto campano per la storia della Resistenza, Napoli, 2010 
[14] Si veda per questo l'intervento di Chiara Volpato.

[15] P.P. Portinaro, Genocidio, in Id., I concetti del male, Torino, Einaudi, 2002; M. Flores, Tutta la violenza di un secolo, Feltrinelli, Milano, 2005. P.P. Portinaro, L'imperativo di uccidere. Genocidio e democidio nella storia, Laterza, Roba-Bari, 2017.

[16] E. Manera, Tempo di mitocrazie. Sulla crisi del presente, in Laboratorio sulle inquietudini e le violenze nel tempo presente: https://fondazionefeltrinelli.it/tempo-di-mitocrazie-sulla-crisi-delpresente/

[17] C. Vercelli, Neofascismo in grigio. La destra radicale tra l'Italia e l'Europa, Einaudi, Torino 2021

[18] M. Aime, Classificare, separare, escludere. Razzismi e identità, Einaudi, Torino 2020

[19] C. Spottorno e G. Abril, La crepa, Add, Torino, 2017; cfr. B. Tertrais e D. Papin, Atlante delle frontiere e delle migrazioni, Add, Torino, 2018; cfr.

[20] E. Traverso, I nuovi volti del fascismo, Ombre corte, Verona, 2017; F. Filippi, Mussolini ha fatto anche cose buone, Bollati Bornghieri, Torino, 2019; C. Greppi, L'antifascismo non serve più a niente, Laterza, Roma-Bari, 2020.

[21] E. Manera, Un'altra storia? Conversazione con Igiaba Scego e Carlo Greppi, in «Doppiozero.com»: https://www.doppiozero.com/materiali/unaltra-storia-conversazione-conigiaba-scego-e-carlo-greppi 